\title{
Clinical variation: why it matters
}

\author{
Anne Duggan', Elizabeth Koff ${ }^{2}$, Villis Marshall ${ }^{1}$
}

Understanding variation in care is an important step in improving patient health outcomes through appropriate care

$\mathrm{V}$ ariation in health care usage is a troubling feature of contemporary medicine. For similar populations, usage of medications, interventions and procedures may be very different and lead to variation in outcomes. The following articles discuss why exploring unwarranted variation is a priority for health care systems; how atlases of health care variation act as catalysts of change and what health care systems can do to increase appropriate care. In this context, the Australian health care systems' response to the problem of unwarranted health care variation is considered.

In November 2015, the Hon Sussan Ley, federal Minister for Health, launched the Australian atlas of healthcare variation. ${ }^{1}$ The Atlas, the first in a series, looks at variation in health care use at population level for 36 clinical activities including medication dispensing, diagnostic and surgical interventions and interventions for chronic diseases. International data, where available, are included and provide context. For example, Australia's rate of dispensing of antibiotics is almost double that of Canada. This variation is consistent with a large body of evidence including that from a recent Organisation for Economic Co-operation and Development report, which included Australian data. ${ }^{2,3}$

In a number of countries, variation is being explored as a way to define and promote appropriate care and thereby value in the health care system. In this Supplement, DaSilva and Gray argue that atlases of clinical variation are catalysts for change by stimulating debate, engaging all participants in the health system so that patient-focused care can be achieved. ${ }^{4}$ The authors argue that no one group has all the necessary information, knowledge or capacity to plan and deliver appropriate care. For this reason, the Australian Commission on Safety and Quality in Health Care (the Commission) and the National Health Performance Authority developed the Australian atlas with input from the states and territories, and clinician and consumer groups. The Atlas's recommendations require action from many parts of the health care system.

Variation is not bad per se. As Buchan and colleagues note, some variation in health care delivery is warranted and desirable, such as meeting differences in patients' health needs or health preferences. ${ }^{2}$ For example, patients with knee pain from osteoarthritis may choose physiotherapy, knee replacement or lifestyle changes. The focus must be on variation that is inexplicable by either patient need or preference and is therefore unwarranted, such as would occur in the above example if treatment options were not offered. Unwarranted variation means people are exposed to real harm from not receiving care that they need or potential harm from receiving care that they do not need and cannot benefit them.

Atlases as such do not demonstrate the impact of unwarranted variation on patient outcomes. Atlases highlight variation such as, in this case, the seven-fold variation in dispensing rates of antipsychotics in people aged over 65 years. Clinical experts, for example, can analyse the data for unwarranted variation, distil the evidence for change into a clinical care standard and thereby effect widespread reduction in unwarranted variation and increase appropriate care. $^{5}$

Research literature often focuses on the harms of not receiving evidence-based care. However, there is increasing awareness that people can be harmed from overdiagnosis and overtreatment, an example being antibiotic treatment of all infections. "Winding back the harms of too much medicine" (http://www. preventingoverdiagnosis.net) and "choosing wisely" (http://www. choosingwisely.org.au) are two recent catchphrases.

In the era of evidence-based medicine, defining what is right should be easier but remains challenging. Evidence of effectiveness is limited and can change over time, particularly for emerging technology, pharmaceuticals and patients with multiple comorbidities. Where evidence exists, implementation science needs improving. Glasziou, a recognised evidence-based medicine expert, describes a leaking evidence-to-practice pipeline. ${ }^{7}$ For evidence-based care to occur, evidence needs to be available, applicable and accepted, and able to be implemented, acted upon and agreed to by the patient. Achieving this requires multiple strategies; organisational prioritisation of the issue as important; resources to achieve the objective; systems to support clinician and consumer adoption of best practice; outcome measurement; and feedback for improvement, as Turnidge and colleagues describe in relation to antimicrobial use in Australian hospitals. ${ }^{8}$ The Commission's work to support clinician and consumer adoption of best practice includes developing clinical care standards, clinical quality registries and patient-reported outcome measures (PROMs). Articles in this Supplement discuss the emerging evidence for these initiatives. ${ }^{9,10}$ For example, mechanisms to measure patient outcomes such as PROMs can assess the effectiveness of care. ${ }^{10,11}$ Clinical quality registries can improve clinical appropriateness and clinical care standards reduce unwarranted variation in treatment, such as for acute coronary syndromes., ${ }^{9,10}$

There is urgency for improving appropriate care because of increasing demand for services from an ageing population, medical science developments and cost escalators. ${ }^{4}$ Mechanisms to define, communicate and continually measure value to the patient may improve patient outcomes, and thereby increase the appropriateness of individual care and the sustainability of health care for populations. Future atlases that provide time-trended data will help. Exploring variation is the first step.

Competing interests: No relevant disclosures.

Provenance: Commissioned; externally peer reviewed.

(c) 2016 AMPCo Pty Ltd. Produced with Elsevier B.V. All rights reserved.

1 Australian Commission on Safety and Quality in Health Care, National Health Performance Authority. Australian atlas of healthcare variation. Sydney: ACSQHC, 2015. http://www.safetyandquality.gov.au/atlas (accessed Oct 2016).

2 Buchan HA, Duggan A, Hargreaves J, et al. Health care variation: time to act. Med J Aust 2016; 205 (10 Suppl): S30-S33. 
3 Australian Commission on Safety and Quality in Health Care, Australian Institute for Health and Welfare. Exploring healthcare variation in Australia: analyses resulting from an OECD study. Sydney: ACSHQC. 2014. http://www.safetyandquality.gov.au/ publications/exploring-healthcare-variation-in-australia (accessed Oct 2016).

4 Da Silva P, Gray JAM. English lessons: can publishing an atlas of variation stimulate the discussion on appropriateness of care? Med J Aust 2016; 205 (10 Suppl): S5-S7.

5 Caplan GA, Kurrle SE, Cumming A. Appropriate care for older people with cognitive impairment in hospital. Med J Aust 2016; 205 (10 Suppl): S12-S15.

6 Welch HG, Schwartz L, Woloshin S. Overdiagnosed: making people sick in the pursuit of health. Beacon Press 2011 .
7 Glasziou P, Haynes B. The paths from research to improved health outcomes. Evid Based Med 2005; 10: 4-7.

8 Turnidge JD, Thursky K, Chen CS, et al. Antimicrobial use in Australian hospitals: how much and how appropriate? Med J Aust 2016; 205 (10 Suppl): S16-S20.

9 Chew DP, Herkes R, Page MA. Clinical care standards: appropriate care everywhere acute coronary syndromes as an example. Med J Aust 2016; 205 (10 Suppl): S8-S11.

10 Wilcox N, McNeil JJ. Clinical quality registries have the potential to drive improvements in the appropriateness of care. Med J Aust 2016; 205 (10 Suppl): S21-S26.

11 Lee $\mathrm{E}$, Emanuel $\mathrm{E}$. Shared decision making to improve care and reduce costs. N Engl J Med 2013; 368: 6-7. 